\title{
Terephthalic Acid Synthesis at Higher Concentrations in High-Temperature Liquid Water. 2. Eliminating Undesired Byproducts
}

\author{
Mitsumasa Osada \\ Dept. of Chemical Engineering, Ichinoseki National College of Technology, Takanashi, \\ Hagisho, Ichinoseki, Iwate 021-8511, Japan \\ Phillip E. Savage \\ Dept. of Chemical Engineering, University of Michigan, Ann Arbor, MI 48109
}

DOI 10.1002/aic.11761

Published online May 5, 2009 in Wiley InterScience (www.interscience.wiley.com).

\begin{abstract}
We synthesized terephthalic acid (TPA) from p-xylene at an initial concentration above its solubility limit in high-temperature liquid water (HTW). The nominal p-xylene loading at the reaction conditions was $0.4 \mathrm{~mol} \mathrm{~L}^{-1}$, which is the highest reported to date for generation of high TPA yields (>70 mol \%) in HTW. The presence of two liquid phases during the reaction did not appear to accelerate the rate, unlike behavior reported for some other organic reactions done "on water" at lower temperatures. Adding oxygen gas in a large increment during synthesis produced a black liquid and a black solid byproduct, which is a previously undocumented problem. Adding oxygen in smaller increments prevented formation of the liquid and solid byproducts and also provided high selectivities (90 mol \%) and yields (>70 mol \%) of TPA. These results demonstrate the feasibility of HTW as a medium for TPA synthesis at p-xylene concentrations even higher than its solubility limit. @ 2009 American Institute of Chemical Engineers AIChE J, 55: 1530-1537, 2009

Keywords: catalysis, supercritical processes, environmental engineering
\end{abstract}

\section{Introduction}

High-temperature liquid water (HTW) is attractive as a reaction medium for organic chemical synthesis because water is inexpensive, abundant, nontoxic, environmentally benign, and because it has desirable chemical properties. ${ }^{1,2}$ A particularly suitable large scale industrial reaction system that might be amenable to a HTW process is $p$-xylene oxidation to terephthalic acid (TPA). ${ }^{3,4}$ TPA is produced commercially in very large quantities by the homogeneously catalyzed selective oxidation of $p$-xylene in acetic acid. The economic and environmental benefits that could result from replacing acetic acid with HTW provided the motivation for this investigation.

\footnotetext{
Correspondence concerning this article should be addressed to P. E. Savage at psavage@umich.edu

(C) 2009 American Institute of Chemical Engineers
}

The technical feasibility of using HTW as a medium for TPA synthesis has been demonstrated..$^{5-13}$ Our group has reported previously on $p$-xylene partial oxidation in HTW, and we obtained high TPA yields $(>80 \mathrm{~mol} \%)$ at $300^{\circ} \mathrm{C}$, [pxylene $]_{0}=0.02 \mathrm{~mol} \mathrm{~L}^{-1},\left[\mathrm{O}_{2}\right]_{0}=0.10 \mathrm{~mol} \mathrm{~L}^{-1},\left[\mathrm{MnBr}_{2}\right]=$ $0.007 \mathrm{~mol} \mathrm{~L}^{-1}$, and $t=5-15 \mathrm{~min} .{ }^{5}$ Moreover, under these conditions and $t=15 \mathrm{~min}$, the yields of carbon monoxide and carbon dioxide, which are products from decomposition of the aromatic ring structure of $p$-xylene and decarboxylation of TPA, were below $2 \%$. $\mathrm{MnBr}_{2}$ was the most active catalyst of those we assessed. Hamley et al. conducted $p$-xylene partial oxidation in supercritical water at $400^{\circ} \mathrm{C}$ and $[p \text {-xylene }]_{0}=0.008-$ $0.05 \mathrm{~mol} \mathrm{~L}^{-1}$ by using a flow reactor. They reported TPA yields exceeding $90 \%$ at residence time less than 1 min..$^{10}$

Very recently, we reported on experiments at a higher reactant concentration $\left([p \text {-xylene }]_{0}=0.2 \mathrm{~mol} \mathrm{~L}^{-1}\right)$ that 
produced about $80 \mathrm{~mol} \%$ TPA yield. ${ }^{9}$ In this work, we found that the oxygen feed method significantly affected the final product distribution and reaction rate. Adding oxygen in small increments, quickly and intermittently, was required to achieve high TPA yields.

It would be advantageous to operate this system with even higher $p$-xylene concentrations so more TPA could be produced per unit reactor volume. Therefore, in this present work, we conducted TPA synthesis at twice the loading of $p$-xylene used in the previous work, namely a nominal concentration of $[p \text {-xylene }]_{0}=0.4 \mathrm{~mol} \mathrm{~L}^{-1}$, in $\mathrm{HTW}$ at $300^{\circ} \mathrm{C}$. The $p$-xylene loading used herein exceeds the solubility of $p$-xylene in HTW at $300^{\circ} \mathrm{C}$ so two liquid phases will exist at reaction conditions, one richer in $p$-xylene than the other. ${ }^{14,15}$

There have been recent demonstrations that the rates of several uni- and bimolecular organic chemical reactions are greatly accelerated when carried out heterogeneously in vigorously stirred aqueous suspensions. ${ }^{16-19}$ Narayan et al. conducted two-phase experiments that began with the organic reactants floating as a separate phase on the surface of the liquid water phase. ${ }^{16}$ The reactant(s) floating "on water" at the start of the experiment led the authors to use this term to describe reactions run in this way. The two-phase mixture is then vigorously stirred and the organic reaction takes place. Doing the reactions "on water" led to rates that were, in some cases, orders of magnitude faster than doing them at the same concentration in a single homogeneous fluid phase. These studies also indicated that both water and fluid phase heterogeneity are required to obtain the accelerated rates. To date "on water" reactions have been limited to temperatures between room temperature and the normal boiling point of water. We are aware of no previous attempts to do organic chemistry "on water" in HTW. The work presented herein represents the first such attempt. In this article, we report on $p$-xylene partial oxidation "on water" in $\mathrm{HTW}$ at $300^{\circ} \mathrm{C}$, where two liquid phases exist at reaction conditions.

\section{Experimental Section}

We purchased all chemicals from Sigma-Aldrich in high purity and used them as received. All experiments were conducted in a $440 \mathrm{~mL}$ Hastelloy FC series autoclave batch reactor fabricated by Pressure Products Industries. (Warminster, PA). The reactor is equipped with a stirrer, dip tube for sampling, and a separate tube for addition of oxygen gas. This latter tube was connected to a syringe pump (Isco model 260D), which enables us to control and alter how ox- ygen gas is added into the preheated HTW reaction medium. The reactor has instrumentation for measuring temperature and pressure.

An experiment began with loading distilled, deionized water $(260 \mathrm{~mL}$ at room temperature), p-xylene $(20 \mathrm{~mL})$, and carefully measured amounts of $\mathrm{MnBr}_{2}$ catalyst into the reactor. We added enough water and $p$-xylene such that the liquid phase occupied $\sim 90 \%$ of the reactor volume at the reaction temperature of $300^{\circ} \mathrm{C}$. p-Xylene solubility in HTW at $294.9^{\circ} \mathrm{C}$ has been reported as 0.0448 (g p-xylene/g water), which is below the nominal concentration of 0.0638 (g p-xylene/g water) used in this work. ${ }^{14}$ Therefore, we expect two separate liquid phases to be present in the reactor at reaction conditions (Unless the presence of the $\mathrm{MnBr}_{2}$ catalyst significantly alters the solubility of $p$-xylene in water). This expectation is consistent with the results of a phase equilibrium calculation that used a modified form of the Soave-Redlich-Kwong equation of state (SRK-EOS) with an exponent-type mixing rule. ${ }^{15}$ The binary interaction parameters for $p$-xylene and water had been previously determined from experimental data by the group at Kyushu University. ${ }^{15}$

After the reactor had been loaded with water, $p$-xylene, and $\mathrm{MnBr}_{2}$ catalyst, it was sealed by bolting the base to the head. Next we began heating and stirring (at $840 \mathrm{rpm}$ ). Pure oxygen from a gas cylinder was fed to the syringe pump and taken to a pressure exceeding that in the reactor. The introduction of oxygen into the reactor defined $t=0$ for the reaction. We added oxygen gas several times throughout the reaction, controlling the amount added and the speed of addition by programming the syringe pump. Oxygen addition was done in this manner for safety purposes. We desired to avoid reactor conditions with large amounts of both $p$-xylene and oxygen present simultaneously, and we also desired to keep the system pressure well below the rated pressure of the autoclave. As the reaction progressed, we withdrew samples periodically from the liquid phase into the sample bulbs. After the reaction, the autoclave reactor was cooled to ambient temperature. We then opened the reactor and recovered the products in the reactor by using dimethyl sulfoxide (DMSO) solvent. We also recovered the products in the sample bulbs. The procedures we followed for batch reactor operation, reactor sampling, and analysis of the products are identical to those described previously. ${ }^{9}$

The aromatic ring balance, selectivity, and product yield are defined as:

aromatic ring balance $($ mol $\%)=\frac{\text { sum of moles of aromatic compounds recovered from the autoclave and from the sample bulbs }}{\text { moles of } p \text {-xylene loaded into the autoclave }}$

selectivity $($ mol $\%)=\frac{\text { moles of product } i(\text { in autoclave or sample bulb })}{\text { sum of moles of all DMSO-soluble organic products }(\text { in autoclave or sample bulb })} \times 100$

$$
\text { product yield }(\text { mol } \%)=\frac{\text { moles of product } i \text { recovered from autoclave after reaction }}{\text { moles } p \text {-xylene loaded in autoclave }- \text { moles aromatic compounds in sample bulbs }} \times 100
$$


Table 1. Product Selectivity and Yields Observed in the Autoclave Reactor After Reaction*

\begin{tabular}{|c|c|c|c|c|c|c|c|c|c|}
\hline \multirow[b]{2}{*}{ Run } & \multicolumn{3}{|c|}{ Conditions } & \multirow[b]{2}{*}{$\begin{array}{c}\text { Aromatic ring } \\
\text { balance }(\mathrm{mol} \%)\end{array}$} & \multicolumn{5}{|c|}{ selectivity $(\mathrm{mol} \%)$ (product yield $(\mathrm{mol} \%))$} \\
\hline & $\mathrm{O}_{2}$ step $^{\dagger}$ & $\begin{array}{c}\text { Final } \mathrm{O}_{2} / p \text {-xylene } \\
(\mathrm{mol} / \mathrm{mol})\end{array}$ & $\begin{array}{c}\mathrm{MnBr}_{2} \\
\left(\mathrm{~mol} \mathrm{~L}^{-1}\right)\end{array}$ & & TPA & $p$-xylene & $\begin{array}{l}p \text {-tolu } \\
\text { aldehyde }\end{array}$ & $\begin{array}{l}p \text {-toluic } \\
\text { acid }\end{array}$ & 4-CBA \\
\hline 1 & 1 & 6 & 0.007 & 28.9 & $99.6(23.1)$ & $0.0(0.0)$ & $0.4(0.1)$ & $0.0(0.0)$ & $0.0(0.0)$ \\
\hline 2 & $0.33,1$ & 4 & 0.007 & 59.6 & $58.4(33.3)$ & $0.0(0.0)$ & $0.0(0.0)$ & $29.4(16.8)$ & $12.2(7.0)$ \\
\hline 3 & 0.33 & 5 & 0.007 & 77.3 & $24.6(18.7)$ & $0.0(0.0)$ & $4.7(3.6)$ & $54.0(41.0)$ & $16.7(12.7)$ \\
\hline 4 & 0.33 & 7 & 0.028 & 80.1 & $90.0(70.9)$ & $0.0(0.0)$ & $2.8(2.2)$ & $7.2(5.7)$ & $0.0(0.0)$ \\
\hline
\end{tabular}

*Reaction condition; $p$-xylene loading $=0.4 \mathrm{~mol} \mathrm{~L}^{-1}$.

The molar ratio of $\mathrm{O}_{2}$ added in a step relative to $p$-xylene initially loaded. The stoichiometric amount needed to convert $p$-xylene to TPA is 3 .

\section{Results}

Table 1 provides a summary of the experimental conditions and the results, based upon the material recovered postreaction from the autoclave reactor. As described below in detail, the reaction time of each run is different and is from 90 to $190 \mathrm{~min}$. All runs were done at a nominal temperature of $300^{\circ} \mathrm{C}$ and with pure oxygen as the oxidant. Table 1 shows that the main products were TPA, $p$-tolualdehyde, $p$ toluic acid, and 4-carboxybenzaldehyde (4-CBA). We also analyzed for 4-methyl benzyl alcohol, 4-hydroxymethyl benzoic acid, terephthaldicarboxaldehyde, phenol, and benzoic acid, but none of these products were detected in this work. All runs shown in Table 1 were carried out once, but our previous research with the same reactor suggests that experimental reproducibility is probably very good..$^{9}$ The aromatic ring balance for each run was between 29 and $80 \mathrm{~mol} \%$. The loss of aromatic rings in Runs 1 and 2 is probably due mainly to the formation of DMSO-insoluble products, which will be described below in detail. In Runs 3 and 4, the loss of aromatic rings was much smaller, and it is probably due to the formation of gas-phase products (e.g., $\mathrm{CO}, \mathrm{CO}_{2}$ ) and the inability to recover all of the water-insoluble solid products such as TPA. The reactor internals had many irregular surfaces which made it difficult to recover completely all of the solids adhering to them.

In Run 1, oxygen was fed to the reactor in discrete bursts. This method is the one we found to be effective for TPA synthesis at a $p$-xylene concentration of $0.2 \mathrm{~mol} \mathrm{~L}^{-1}$, which was a single liquid-phase reaction system. ${ }^{9}$ Each burst of oxygen in Run 1 contained 0.162 moles, which is equal to the number of moles of $p$-xylene initially loaded into the reactor. Thus, the step size for the $\mathrm{O}_{2}$ feed increment was $1.0 \mathrm{~mol}$ $\mathrm{O}_{2} /$ mol $p$-xylene, as shown in Figure 1b. The final molar $\mathrm{O}_{2} /$ $p$-xylene ratio was 6 . The stoichiometric amount needed to convert $p$-xylene to TPA is 3 .

When the sample bulbs from Run 1 were opened and the material recovered by DMSO additions, a black DMSO-insoluble solid product was observed. The black solid product was present even after the first oxygen injection was done at $t=0$ and it was present in all subsequent sample bulbs after $t=0$. The black solid was absent in the sample bulbs filled before oxygen was introduced into the reactor. The formation of the black solid product might be due to oligomerization reactions involving $p$-xylene and/or intermediate oxidation products at this high $p$-xylene loading reaction condition. This experimental condition is the highest $p$-xylene concentration used to date for TPA synthesis in HTW, and there have been no prior reports of the formation of black material or DMSO-insoluble products. Thus, this problem is a new one, which surfaced in this experiment conducted above the $p$-xylene solubility limit in HTW, where two separate liquid phases coexist.

We transferred all of the material recovered from the sample bulbs into $10 \mathrm{~mL}$ flasks. Particles of the black solid product floated in the flask at first, but they settled to the bottom one day later. Since the solid particles did not settle immediately, they are probably not $\mathrm{MnO}_{2}$, which could have
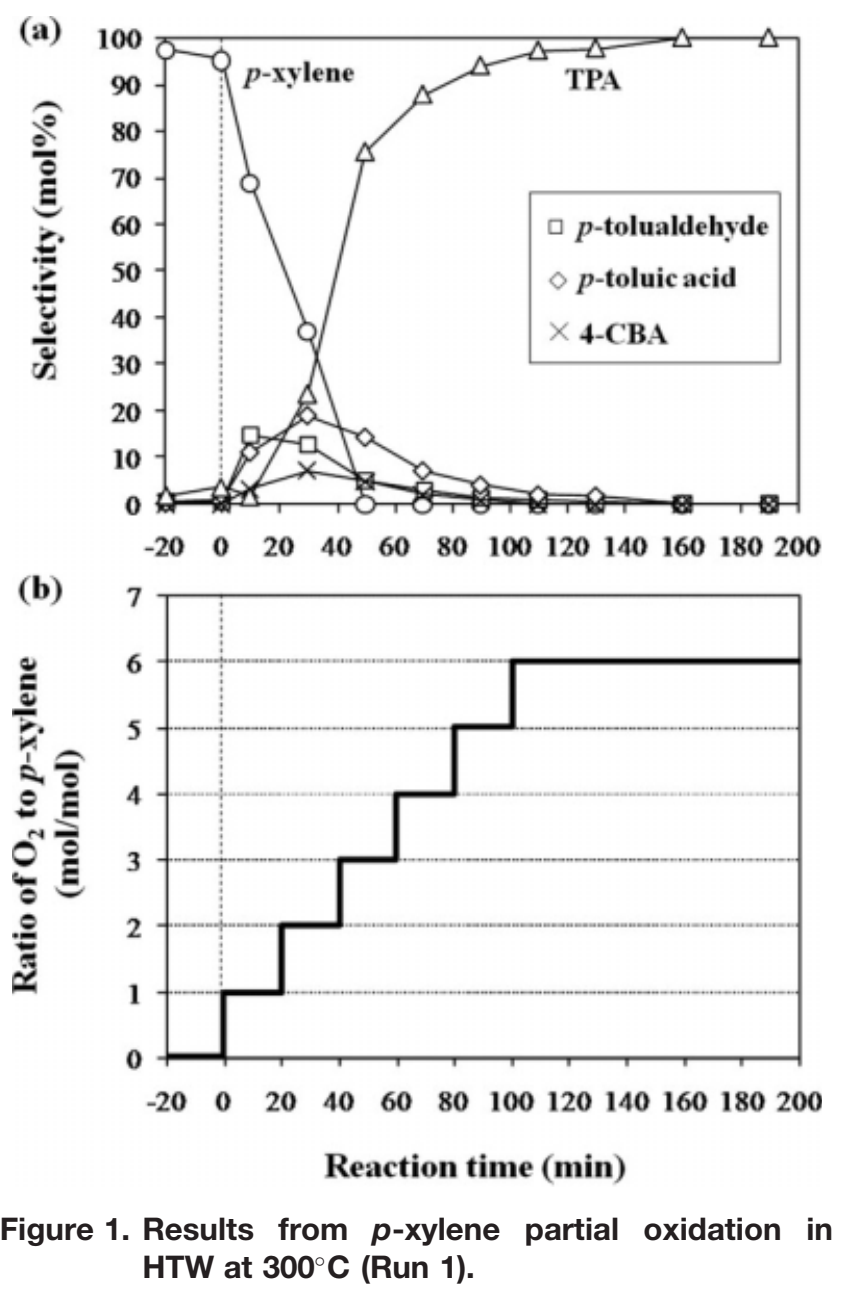

(a) product selectivity; (b) oxygen feed program. 
been formed from oxidation of the Mn catalyst. $\mathrm{MnO}_{2}$ has a much higher density than DMSO. We analyzed the supernatant liquid phase in the flask by HPLC. Figure 1a displays the temporal variation of the product selectivity, as determined from analysis of the sample bulbs. These product selectivities do not include the contribution from the black solid product. In Figure 1a, $p$-xylene disappeared gradually and $p$-tolualdehyde was the product present in the highest selectivity at about $5 \mathrm{~min}$. The $p$-tolualdehyde selectivity then decreased as it was oxidized to $p$-toluic acid, which subsequently formed 4-CBA. The selectivity of $p$-toluic acid and 4-CBA increased to a maximum around $30 \mathrm{~min}$ and then decreased as these products were oxidized further to TPA. The highest TPA selectivity was essentially $100 \mathrm{~mol} \%$. Note that $100 \%$ selectivity does not imply $100 \%$ yield. Rather, it simply implies that by the end of the run, the $p$ xylene and intermediate oxidation products had all reacted completely to form either TPA or the black solids.

We recovered the water-soluble and water-insoluble products from the batch reactor after the reaction. Figure 2 shows

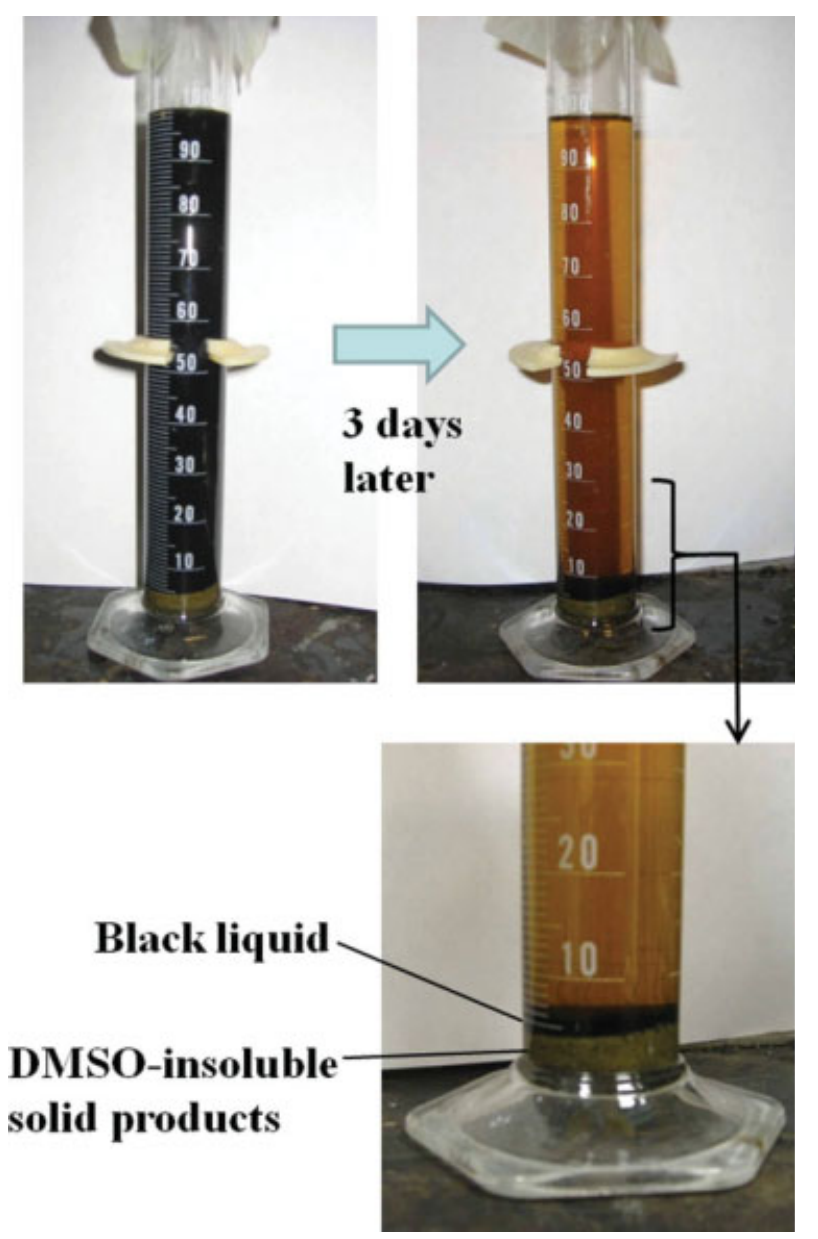

Figure 2. DMSO solution recovered from the autoclave reactor (Run 1).

Note black liquid layer and DMSO-insoluble solids at bottom of graduated cylinder. [Color figure can be viewed in the online issue, which is available at www.interscience.wiley. com.] images of the water-insoluble products recovered by using DMSO solvent. At first, the color of the DMSO solution was black, and there was a DMSO-insoluble solid product in the bottom of the cylinder. We suspect that these solids contain primarily organic material because there was not enough $\mathrm{Mn}$ in the reactor for the solids to be $\mathrm{MnO}_{2}$. Even if all of the $\mathrm{Mn}$ in the reactor were oxidized and precipitated as $\mathrm{MnO}_{2}$, it would occupy only about $0.05 \mathrm{~cm}^{3}$. The volume of the solids layer in Figure 2, on the other hand, is about 100 times larger. Three days later, the color of the DMSO solution in the graduated cylinder became yellow, and a black liquid layer and a separate DMSO-insoluble solid layer were observed in the bottom of the cylinder. We did not analyze the black liquid or the solids because we were less interested in characterizing these undesired byproducts than in finding synthesis conditions that prevented their formation. We did analyze the yellow supernatant liquid phase by HPLC.

In Table 1 , the aromatic ring balance for Run 1 is only 29 mol\%, which is consistent with a large fraction of the $p$-xylene loaded into the reactor being converted to black liquid and a DMSO-insoluble solid byproduct. The product selectivities obtained from analysis of the postreaction material recovered from the autoclave (Table 1) are comparable to those obtained from analysis of the final sample bulbs (Figure 1). The TPA selectivity was $99.6 \mathrm{~mol} \%$, but its yield was only $23 \mathrm{~mol} \%$.

In Run 1, the formation of the black solid product occurred after the first injection of oxygen. It did not occur before the addition of oxygen. Since no solids were ever observed in our previous study, ${ }^{9}$ which used smaller oxygen addition increments, we reasoned that perhaps adding oxygen in a smaller increment might reduce the propensity for solids formation in the present experiments. In Run 2, we added the first three oxygen bursts in a smaller increment of 0.33 moles of $\mathrm{O}_{2}$ per mole $p$-xylene initially loaded. Subsequent oxygen additions were the same amount used in Run 1. Figure $3 \mathrm{~b}$ shows the complete oxygen addition program. In Run 2, the black solid product was not observed in any of the samples taken through $33 \mathrm{~min}$. Solids were observed, however, in all sample bulbs after $40 \mathrm{~min}$. The oxygen addition step at 40 min was the first of the larger additions in Run 2. The amount added here was the same as that added in each step in Run 1. This result suggests that adding oxygen in this large increment is associated with the formation of DMSO-insoluble products at this high $p$-xylene concentration condition. In addition, it appears that the formation of solids may be preventable by adding oxygen in smaller increments.

Figure 3a shows the temporal variation of the product selectivities from Run 2. As mentioned above, we observed the formation of a black solid product after $40 \mathrm{~min}$ and we analyzed only the supernatant liquid phase by HPLC. Therefore, the product selectivity after $40 \mathrm{~min}$ does not include the black solid product. $p$-Xylene decreased gradually for the first $30 \mathrm{~min}$ and then after that its rate of disappearance increased dramatically. $p$-Tolualdehyde was the product present in the highest selectivity for the first $50 \mathrm{~min}$. The $p$-tolualdehyde selectivity then decreased as this product was oxidized to $p$-toluic acid, which subsequently formed 4-CBA. The selectivity of $p$-toluic acid and 4-CBA increased to maximum values at around $70 \mathrm{~min}$ and then decreased as further 

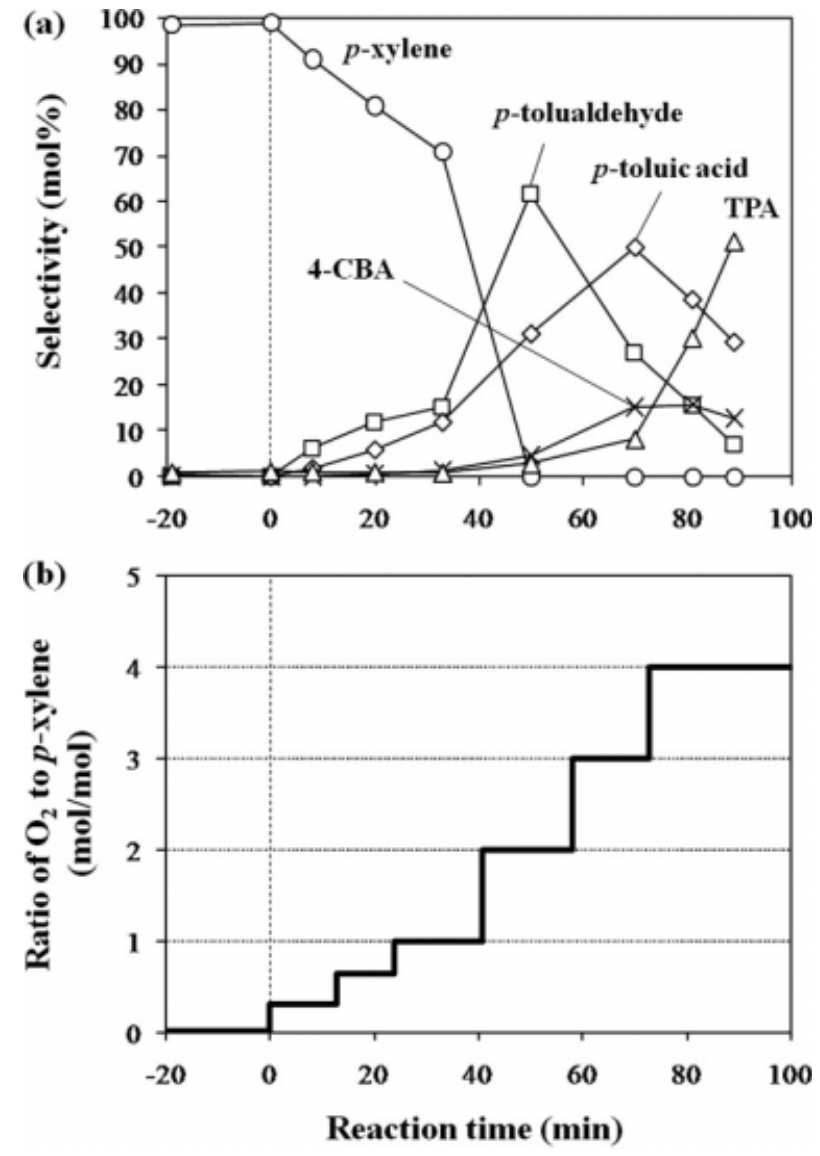

Figure 3. Results from $p$-xylene partial oxidation in $\mathrm{HTW}$ at $300^{\circ} \mathrm{C}$ (Run 2).

(a) product selectivity; (b) oxygen feed program.

oxidation to TPA occurred. The highest TPA selectivity was $51 \mathrm{~mol} \%$, but this selectivity was increasing and significant amounts of intermediate oxidation products remained. It is likely that a higher TPA selectivity (as in Run 1) could be attained by extending the reaction time and adding more oxygen. The TPA selectivity from Run 2 obtained from analysis of the batch reactor contents after the reaction was 58 mol \% (Table 1), which is in good correspondence with that of the final sample bulb in Figure 3.

Table 1 shows that the aromatic ring balance for Run 2 was $60 \mathrm{~mol} \%$, indicating that no more than $40 \mathrm{~mol} \%$ or so of the $p$-xylene loaded into the reactor was converted to the black liquid and DMSO-insoluble solid product. The aromatic ring balance of Run 2 was higher than that of Run 1, indicating that the amount of the black liquid and DMSO-insoluble solid product in Run 2 was probably lower than that in Run 1.

From Run 2, we learned that adding oxygen in a smaller increment is effective for preventing the formation of the DMSO-insoluble products. Therefore, we next conducted an experiment (Run 3) with oxygen addition always being in this small increment of 0.33 moles $\mathrm{O}_{2}$ per mole $p$-xylene, as shown in Figure 4. This oxygen addition strategy differs from that in Run 2 in that the larger steps have been omitted and replaced with a larger number of smaller steps. No black liquid or DMSO-insoluble solid material was observed in any of the sample bulbs or in the autoclave reactor after the experiment, indicating that this change in the oxygen feed method was effective for suppressing these reactions under the conditions of Run 3 .

Figure 4 shows the temporal variation of the product selectivity of Run 3. p-Xylene decreased gradually and disappeared at about $80 \mathrm{~min}$. $p$-Tolualdehyde was the product present in the highest selectivity for the first $40 \mathrm{~min}$. Its selectivity decreased after $80 \mathrm{~min}$ as it was oxidized to $p$-toluic acid, which subsequently formed 4-CBA. The selectivities of p-toluic acid, 4-CBA, and TPA increased gradually throughout the time period examined. The highest TPA selectivity was $21.7 \mathrm{~mol} \%$.

In Table 1, the aromatic ring balance from Run 3 was 77 mol \%. There was no black liquid and DMSO-insoluble solid product in the material remaining in the autoclave. Therefore, the loss of aromatic rings is probably due to the formation of gas products (e.g., $\mathrm{CO}, \mathrm{CO}_{2}$ ) and the inability to recover all of the water-insoluble solid products such as TPA. The TPA selectivity of Run 3 obtained from the batch reactor after the reaction was $24.6 \mathrm{~mol} \%$, which is in good correspondence with that of the sample bulbs in Figure 4.
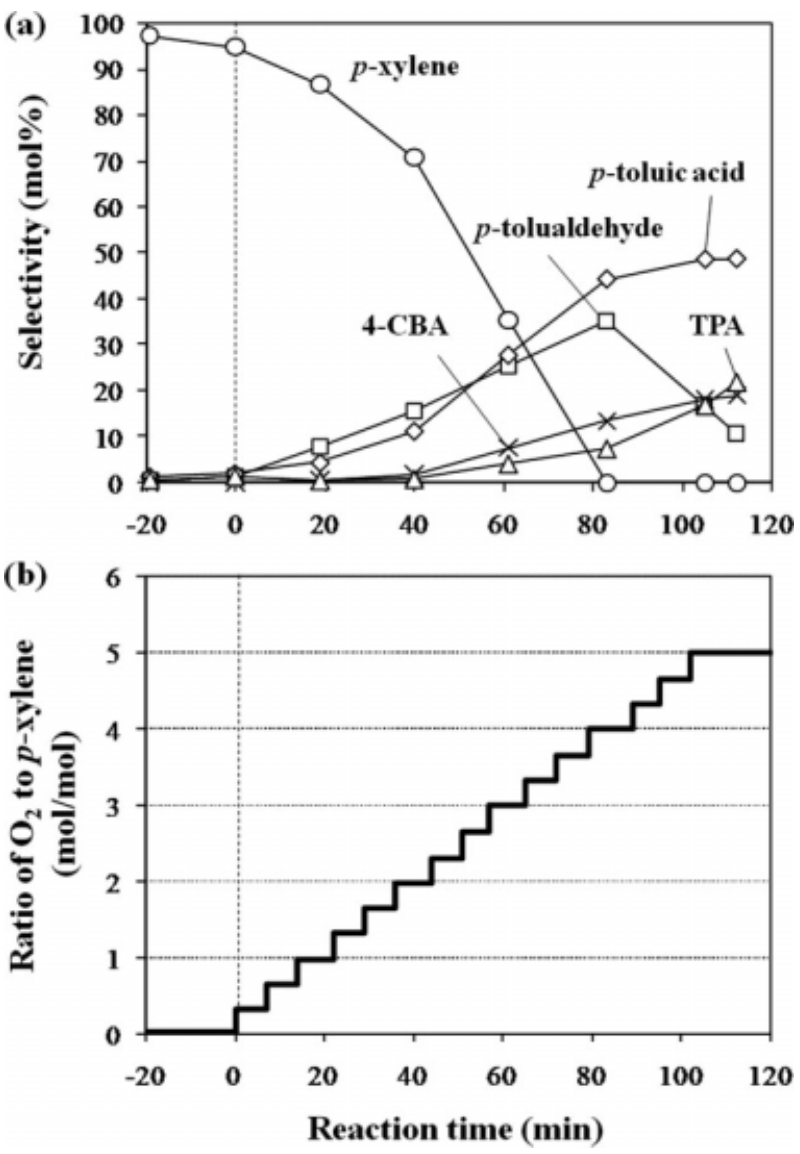

Figure 4. Results from $p$-xylene partial oxidation in HTW at $300^{\circ} \mathrm{C}$ (Run 3).

(a) product selectivity; (b) oxygen feed program. 
The TPA selectivity from Run 3 is lower than that from Runs 1 and 2, and the selectivities of $p$-tolualdehyde, p-toluic acid and 4-CBA from Run 3 are higher than those from Runs 1 and 2. This result indicates that the partial oxidation reaction from $p$-xylene to TPA did not proceed as far as in Run 3, even though the formation of black liquid and DMSO-insolubles was suppressed.

In Run 3, we obtained a high aromatic ring balance, but the selectivity and yield of TPA were low. We hypothesized that increasing the catalyst and oxygen loadings, while maintaining the small oxygen addition increments, might allow us to achieve both high aromatic ring balances (e.g., suppress liquid and DMSO-insolubles formation) and high TPA selectivity. Run 4 used an ultimate $\mathrm{O}_{2} / p$-xylene ration of 7 , a longer reaction time of $160 \mathrm{~min}$, and four times greater amount of catalyst than that of Run 3. The increment of oxygen added in each burst was 0.33 moles $\mathrm{O}_{2}$ per mole $p$-xylene, which is the same as that in Run 3. Figure 5b shows the oxygen addition profile for this experiment. In Run 4, no black liquid or DMSO-insoluble solid product was observed in any of the sample bulbs or in the batch reactor after the experiment.

Figure 5a shows the temporal variation of the product selectivity of Run 4. p-Xylene had disappeared by $60 \mathrm{~min}$.
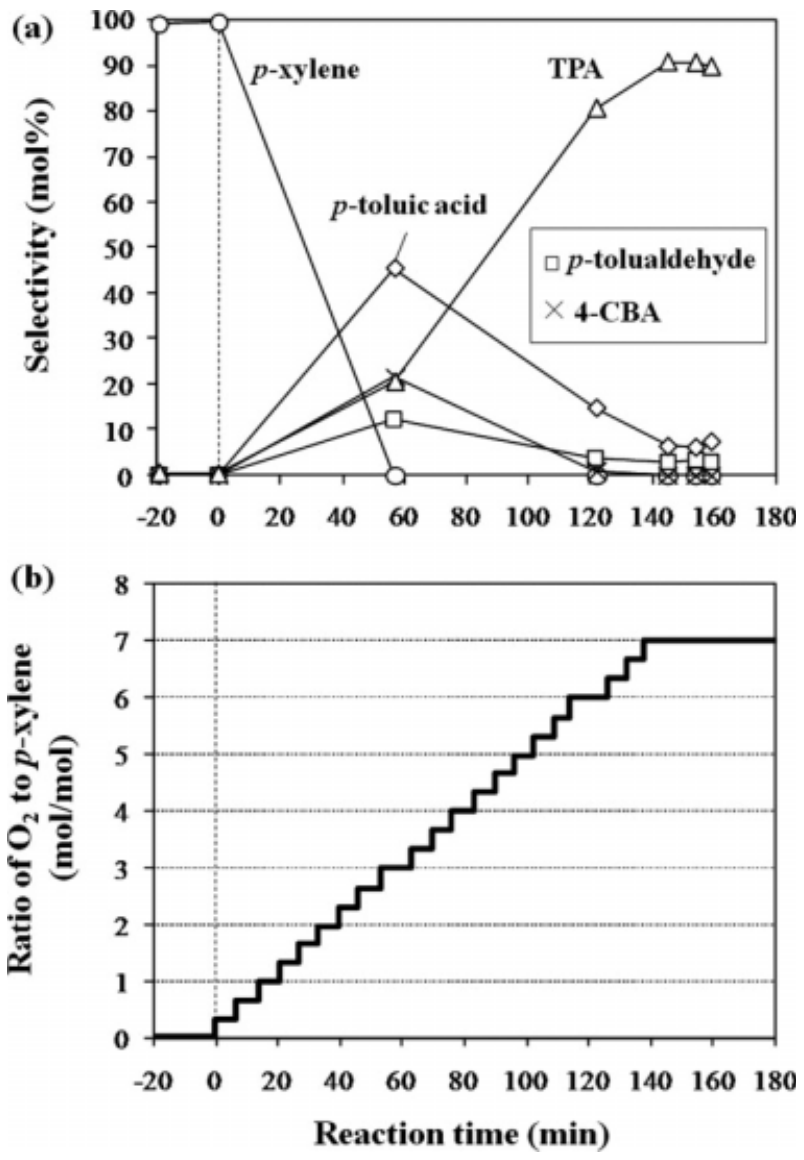

Figure 5. Results from $p$-xylene partial oxidation in HTW at $300^{\circ} \mathrm{C}$ (Run 4).

(a) product selectivity; (b) oxygen feed program.
p-Tolualdehyde, $p$-toluic acid, and 4-CBA were the partial oxidation products present in the highest selectivity at about $60 \mathrm{~min}$. These selectivities decreased at longer times. The selectivity of TPA gradually increased and reached $90 \mathrm{~mol} \%$ at around $140 \mathrm{~min}$.

In Table 1, the aromatic ring balance for Run 4 was 80.1 mol \%, which is almost the same as that from Run 3. The postreaction TPA selectivity of Run 4 was 90 mol \%, which again is in good correspondence with that of the final sample bulb in Figure 5. In Run 4, we achieved both a high aromatic ring balance and a high TPA selectivity and yield from $p$-xylene oxidation at a high initial loading.

\section{Discussion}

\section{"On water" in HTW}

In this work, we conducted experiments at $p$-xylene loadings that exceededits solubility limit in $\mathrm{HTW}$ at $300^{\circ} \mathrm{C}$. Thus, two separate liquid phases were likely present at reaction conditions. As noted in the introduction, others have reported that some organic reactions conducted with water in twophase conditions exhibited dramatic acceleration of the reaction rates. ${ }^{16-19}$ We can assess whether this is the case for $p$-xylene oxidation in HTW by comparing the present results with those from previous work ${ }^{5,9}$ at $[p \text {-xylene }]_{0}=0.02$ and $0.2 \mathrm{~mol} \mathrm{~L}^{-1}$. These earlier studies were conducted within the solubility limits of $p$-xylene in water, which means that a single homogeneous liquid phase existed at reaction conditions. Both of these previous experiments used the same batch reactor, but the feed methods for $p$-xylene, catalyst, and oxygen were different. In our previous research at $[p \text {-xylene }]_{0}=0.02$ mol L $\mathrm{L}^{-1}$, we loaded water and air into the batch reactor and heated it to $300^{\circ} \mathrm{C} .^{5}$ At this point, we injected the $p$-xylene and $\mathrm{MnBr}_{2}$ catalyst by the syringe pump. Our other previous study, at $[p \text {-xylene }]_{0}=0.2 \mathrm{~mol} \mathrm{~L}^{-1}$, was conducted with the same feed methods of $p$-xylene, catalyst, and oxygen that were used in this present study. ${ }^{9}$ Although there are some experimental differences, we tried to compare these experiments as follows. In Run 4 (Figure 5), which showed the highest TPA selectivity in this work, the TPA selectivity reached $90 \mathrm{~mol} \%$ at about $140 \mathrm{~min}$. From this result, and assuming that the yield would be comparable to the selectivity if we were able to recover all postreaction material, we evaluated the average reaction rate to be about $2.4 \times 10^{-3}$ mol L ${ }^{-1} \min ^{-1}$. From our previous research at $[p \text {-xylene }]_{0}=$ $0.02 \mathrm{~mol} \mathrm{~L}^{-1}$, it took about $5 \mathrm{~min}$ to reach $90 \mathrm{~mol} \%$ yield of TPA, and the average reaction rate was evaluated as about $3.3 \times 10^{-3} \mathrm{~mol} \mathrm{~L}^{-1} \min ^{-1} .5$ For the case of $[p \text {-xylene }]_{0}=$ $0.2 \mathrm{~mol} \mathrm{~L}^{-1}$, it took about $40 \mathrm{~min}$ to reach $90 \mathrm{~mol} \%$ yield of TPA, so the average reaction rate was about $4.1 \times 10^{-3} \mathrm{~mol}$ $\mathrm{L}^{-1} \min ^{-1}$. 9 These rough calculations indicate that the average reaction rate in the present work, which used two separate liquid phases, was roughly the same as those observed in previous studies, which used a single homogeneous liquid phase. Absent any "on water" effects, one would expect a higher rate in the present study because the concentrations of the reactants and catalyst were higher than in previous work. No higher rate was observed, which indicates that the present study provides no evidence for any rate acceleration in this system that could be attributed to the "on water" effect. We 
do offer a word of caution here, however, since average rates were used in the comparison and since the reaction rate at these higher $p$-xylene concentrations strongly depends on the frequency and amount of oxygen fed in each discrete burst, as noted in our previous research. ${ }^{9}$ Faster average rates could be obtained at the higher $p$-xylene concentrations if the time between oxygen bursts could be reduced. The limiting factor in this time between bursts was the amount of time required to reload the syringe pump with oxygen and then pressurize it before the next injection. This length of time could be reduced by adding a second syringe pump to the experimental system, but we did not have a second pump for this study.

It may also be important to note that the two liquid phases present during our reactions were both richer in water than in $p$-xylene. The phase equilibrium calculations indicated that one phase would be $>99 \mathrm{~mol} \%$ water and the other phase would be about $90 \pm 5 \mathrm{~mol} \%$ water (where the uncertainty is due to uncertainty in the measured system pressure). Both phases being largely aqueous may be an important distinction from the "on water" experiments in the literature at lower temperatures, where the mutual solubilities of the organic compound(s) and water were much lower.

\section{Effect of oxygen addition}

Our previous article on TPA synthesis at high $p$-xylene concentrations $\left([p \text {-xylene }]_{0}=0.2 \mathrm{~mol} \mathrm{~L}^{-1}\right)$ showed that adding oxygen quickly and in small increments is a method for achieving a high selectivity and yield of TPA. ${ }^{9}$ We hypothesized that the quick oxygen injection creates additional mixing and a temporary but instantaneous high local concentration of oxygen in the liquid phase in the vicinity of the dip tube. This high concentration was favorable because oxygen serves as an oxidant for the formation of active catalysis species and as a reactant for the $p$-xylene partial oxidation. ${ }^{9,20}$ The additional insight gained in the present study is that intermittent bursts of oxygen alone are not sufficient for ensuring high TPA yields and selectivities. Indeed, Run 1 used this oxygen feed method and produced primarily a black liquid and DMSO-insoluble solids. The amount of oxygen added in each burst in Run 1, however, was larger than that added in any of the experiments in our previous work. Thus, it appears that the amount of oxygen added per burst is also an important variable for this system. Adding too much oxygen may create a local oxygen concentration that is too high for controllable TPA synthesis, and instead promotes oligomerization reactions of $p$-xylene or intermediate products. Indeed, when we added oxygen in a smaller increment (Run 4), we did not observe the formation of any liquid or black solid products and we obtained a high aromatic ring balance and high TPA selectivity. In this case, the instantaneous oxygen concentration would be smaller than that in Run 1. To achieve high TPA selectivity and avoid forming presumed oligomerization products at high $p$-xylene loadings, an optimization of the amount of oxygen added in each increment is important.

\section{Conclusion}

This work demonstrates that TPA can be formed in high selectivity (>90 mol \%) from $p$-xylene even at high initial loadings $\left(0.4 \mathrm{~mol} \mathrm{~L}^{-1}\right)$ in $\mathrm{HTW}$ at $300^{\circ} \mathrm{C}$. The $p$-xylene loading exceeded its solubility in $\mathrm{HTW}$ at $300^{\circ} \mathrm{C}$, which means that there are two liquid phases at reaction conditions. When we added oxygen in a large increment of 1.0 mole of $\mathrm{O}_{2}$ per mole $p$-xylene loaded, the main products were a black liquid and DMSO-insoluble solids. These undesired byproducts were probably formed through oligomerization reactions between $p$-xylene and/or intermediate products. In contrast, when we added oxygen in a smaller increment of 0.33 moles $\mathrm{O}_{2}$ per mole $p$-xylene, we obtained a high TPA selectivity and suppressed the formation of undesired solid and liquid byproducts. These results suggest that highly selective TPA synthesis may be possible in HTW at even higher $p$-xylene loadings via optimization of the amount of oxygen fed in each burst. We believe that this system holds tremendous promise for the implementation of a green chemistry and engineering approach for production of a bulk commodity chemical.

\section{Acknowledgements}

This research was supported in part by the National Science Foundation (Grant CTS-0625641), the ACS Petroleum Research Fund (45642AC4), and also by the Institute of National Colleges of Technology, Japan. The authors thank Dr. Yoshio Iwai and Dr. Yusuke Shimoyama (both of Kyushu University, Japan) for assistance with the phase equilibria calculations.

\section{Literature Cited}

1. Savage PE. Organic chemical reactions in supercritical water. Chem Rev. 1999;99:603-621.

2. Akiya N, Savage PE. Roles of water for chemical reactions in high temperature water. Chem Rev. 2002;102:2725-2750.

3. Dunn JB, Savage PE. Economic and environmental assessment of high-temperature water as a medium for terephthalic acid synthesis. Green Chem. 2003;5:649-655.

4. Fraga-Dubreuil J, Poliakoff M. Organic reactions in high-temperature and supercritical water. Pure Appl Chem. 2006;78:1971-1982.

5. Dunn JB, Savage PE. High-temperature liquid water: a viable medium for terephthalic acid synthesis. Environ Sci Technol. 2005;39: 5427-5435.

6. Dunn JB, Savage PE. Terephthalic acid synthesis in high-temperature liquid water. Ind Eng Chem Res. 2002;41:4460-4465.

7. Dunn JB, Urquhart DI, Savage PE. Terephthalic acid synthesis in supercritical water. Adv Synth Catal. 2002;344:385-392.

8. Savage PE, Dunn JB, Yu J. Recent advances in catalytic oxidation in supercritical water. Combust Sci Technol. 2006;178:443-465.

9. Osada M, Savage PE. Terephthalic acid synthesis at higher concentrations in high-temperature liquid water. 1. effect of oxygen feed method. AIChE J. 2009;55:710-746.

10. Hamley PA, Ilkenhans T, Webster JM, Garcia-Verdugo E, Venardou E, Clarke MJ, Auerbach R, Thomas WB, Whiston K, Poliakoff M. Selective partial oxidation in supercritical water: the continuous generation of terephthalic acid from para-xylene in high yield. Green Chem. 2002;4:235-238.

11. Garcia-Verdugo E, Venardou E, Thomas WB, Whiston K, Partenheimer W, Hamley PA, Poliakoff M. Is it possible to achieve highly selective oxidations in supercritical water? aerobic oxidation of methylaromatic compounds. Adv Synth Catal. 2004;346:307-316.

12. Garcia-Verdugo E, Fraga-Dubreuil J, Hamley PA, Thomas WB, Whiston K, Poliakoff M. Simultaneous continuous partial oxidation of mixed xylenes in supercritical water. Green Chem. 2005;7:294-300.

13. Holliday RL, Jong BYM, Kolis JW. Organic synthesis in subcritical water oxidation of alkyl aromatics. J Supercrit Fluids. 1998;12:255260.

14. Pryor WA, Jentoft RE. Solubility of $\mathrm{m}$ - and p-xylene in water and in aqueous ammonia from $0^{\circ} \mathrm{C}$ to $300^{\circ} \mathrm{C}$. J Chem Eng Data. 1961;6: 36-37. 
15. Haruki M, Iwai Y, Nagao S, Arai Y. Measurement and correlation of liquid-liquid equilibria for water + aromatic hydrocarbon binary systems at high temperatures and pressures. J Chem Eng Data. 2001;46:950-953.

16. Narayan S, Muldoon J, Finn MG, Fokin VV, Kolb HC, Sharpless KB. "On water": unique reactivity of organic compounds in aqueous suspension. Angew Chem Int Ed. 2005;44:3275-3279.

17. Dambacher J, Zhao W, El-Batta A, Anness R, Jiang C, Bergdahl M. Water is an efficient medium for witting reactions employing stabilized ylides and aldehyde. Tetrahedron Lett. 2005;46:4473-4477.
18. Klijn JE, Engberts BFN. Fast reactions 'on water'. Nature. 2005; 435:746-747.

19. Grieco PA, Brandes EB, McCann SM, Clark JD. Water as a solvent for the claisen rearrangement: practical implications for synthetic organic chemistry. J Org Chem. 1989;54:5849-5851.

20. Chen Y, Fulton JL, Partenheimer W. The structure of the homogeneous oxidation catalyst, $\mathrm{Mn}(\mathrm{II})\left(\mathrm{Br}^{-1}\right)_{\mathrm{X}}$, in supercritical water: an X-ray absorption fine-structure study. J Am Chem Soc. 2005;127:14085-14093.

Manuscript received Aug. 5, 2008, and revision received Oct. 15, 2008. 